\title{
Cooperative AF Relaying in Spectrum-Sharing Systems: Performance Analysis under Average Interference Power Constraints and Nakagami- $m$ Fading
}

\author{
Minghua Xia and Sonia Aïssa, Senior Member, IEEE
}

\begin{abstract}
Since the electromagnetic spectrum resource becomes more and more scarce, improving spectral efficiency is extremely important for the sustainable development of wireless communication systems and services. Integrating cooperative relaying techniques into spectrum-sharing cognitive radio systems sheds new light on higher spectral efficiency. In this paper, we analyze the end-to-end performance of cooperative amplify-andforward (AF) relaying in spectrum-sharing systems. In order to achieve the optimal end-to-end performance, the transmit powers of the secondary source and the relays are optimized with respect to average interference power constraints at primary users and Nakagami- $m$ fading parameters of interference channels (for mathematical tractability, the desired channels from secondary source to relay and from relay to secondary destination are assumed to be subject to Rayleigh fading). Also, both partial and opportunistic relay-selection strategies are exploited to further enhance system performance. Based on the exact distribution functions of the end-to-end signal-to-noise ratio (SNR) obtained herein, the outage probability, average symbol error probability, diversity order, and ergodic capacity of the system under study are analytically investigated. Our results show that system performance is dominated by the resource constraints and it improves slowly with increasing average SNR. Furthermore, larger Nakagami- $m$ fading parameter on interference channels deteriorates system performance slightly. On the other hand, when interference power constraints are stringent, opportunistic relay selection can be exploited to improve system performance significantly. All analytical results are corroborated by simulation results and they are shown to be efficient tools for exact evaluation of system performance.
\end{abstract}

Index Terms-Cognitive radio spectrum sharing, cooperative amplify-and-forward (AF) relaying, interference power constraints, performance analysis, relay selection.

\section{INTRODUCTION}

A $\mathrm{S}$ the applications of wireless communications continue to spread and get more and more diverse, the indispensable electromagnetic spectrum resource becomes more and more scarce and, thus, improving spectrum usage and transmission efficiency is becoming an extremely important topic both in academia and industry. Unlike exclusive utilization of spectrum, spectrum-sharing cognitive radio benefits improving spectral efficiency by sharing spectrum among different users

Paper approved by F. Santucci, the Editor for Wireless System Performance of the IEEE Communications Society. Manuscript received June 25, 2011; revised November 5, 2011 and January 19, 2012.

M. Xia is with the Division of Physical Sciences and Engineering, King Abdullah University of Science and Technology (KAUST), Thuwal, Saudi Arabia (e-mail: minghua.xia@kaust.edu.sa).

S. Aïssa is with INRS, University of Quebec, Montreal, QC, Canada and with KAUST, Thuwal, Saudi Arabia (e-mail: sonia.aissa@ieee.org).

Digital Object Identifier *** and thus it is appealing in practice [1]. Specifically, from the licensee's point of view, primary users originally licensed with spectrum resource can work together with secondary users without explicitly assigned spectrum by sharing the spectrum as long as the interference power from secondary users remains below a tolerable threshold. In general, the interference power threshold can be defined by means of the peak interference power or average interference power or both [2]. The peak interference power constraint is appropriate for real-time traffic, and it requires the instantaneous channel gains of interference channels (i.e., the channels from secondary transmitter to primary receivers) to determine the transmit power of secondary users, yielding high feedback overhead in practical systems. On the other hand, the average interferencepower constraint is applicable to non-real time traffic where the quality-of-service (QoS) depends on the average output signalto-noise ratio (SNR), and it results in less feedback overhead.

Since the transmit power of secondary users is strictly limited in spectrum-sharing systems, cooperative relaying techniques can be further exploited to extend wireless coverage and enhance system performance. Generally, there are two protocols to implement relaying: one is decode-and-forward (DF) protocol and the other is amplify-and-forward (AF) protocol [3]. DF relay decodes the received signal, re-encodes and forwards it to the destination. In other words, the consecutive hops in DF relaying systems are separated by the decoding operation and system performance is dominated by the worst hop. On the other hand, AF relay amplifies the received signal and forwards it to the destination such that each hop contributes to system performance.

Integrating cooperative relaying techniques into spectrumsharing systems sheds new light on higher spectral efficiency. With this regard, the maximum delay-constrained throughput of cooperative DF relaying in spectrum-sharing systems is studied in [4], [5]. The ergodic capacity and outage probability of cooperative DF relaying in spectrum-sharing systems with peak interference power constraint are evaluated in [6], [7], respectively. The end-to-end performance of cooperative DF relaying in spectrum-sharing systems is investigated in [8], [9]. A visible drawback of DF relay in spectrum-sharing systems is that system performance is dominated by the worst hop between source-relay and relay-destination hops. Therefore, it is usually assumed that the DF relaying node is deployed approximately in the middle between the secondary source and its destination node [2], [7], which is not necessarily the case in practice since the users in real systems are generally randomly distributed in geography. Moreover, for 
the DF technique, when there are multiple relaying nodes, only partial relay selection can be applied. That is, relay selection can only be performed at either the source-relay hop or the relay-destination hop. Clearly, the chosen "best" relay at one hop cannot guarantee the quality of channel at the other hop. Therefore, partial relay selection is suboptimal when the end-to-end (i.e., from secondary source to its destination via intermediate relay) performance is considered.

In this paper, we investigate the end-to-end performance of cooperative AF relaying in spectrum-sharing systems. In the system, secondary users and intermediate relays share the spectrum originally licensed to primary users, and the transmit powers of secondary source and relays are strictly limited by the average tolerable interference powers at primary users. In order to study the effect of interference channels on the end-to-end performance, interference channels are assumed to be subject to Nakagami- $m$ fading, while Rayleigh fading is extensively supposed in the literature [5], [9]. Therefore, the transmit powers of secondary source and relays are optimized with respect to the average interference power constraints at primary users and the Nakagami- $m$ fading parameters of interference channels (for mathematical tractability, the desired channels from secondary source to relay and from relay to secondary destination are assumed to be subject to Rayleigh fading). Moreover, two relay-selection strategies including partial relay selection and opportunistic relay selection are exploited to further enhance system performance. Based on optimal transmit power allocation and relay selection, exact expressions for the end-to-end SNR are explicitly derived. Subsequently, system performance is investigated considering important performance metrics, namely, outage probability, average symbol error probability, diversity order, and ergodic capacity. Our results show that system performance is dominated by the average interference power constraints and it improves slowly with increasing average SNR. Furthermore, larger Nakagami- $m$ fading parameter of interference channels deteriorates system performance slightly. On the other hand, when the average tolerable interference powers are stringent, the opportunistic relay-selection strategy can be exploited to improve system performance significantly.

The rest of this paper is organized as follows. Section II details the system model. Section III discusses the criteria for optimal power allocation and relay selection. The distribution functions of the end-to-end SNR are derived in Section IV. The performance analysis is conducted in Section V and, finally, some concluding remarks are provided in Section VI.

\section{SYSTEM MODEL}

We consider a spectrum-sharing cooperative AF relaying system as shown in Fig. 1, where two secondary users $\left(\mathrm{SU}_{1}\right.$ and $\mathrm{SU}_{2}$ ) are located in the vicinity of two primary users $\left(\mathrm{PU}_{1}\right.$ and $\left.\mathrm{PU}_{2}\right)$, respectively. The secondary source $\mathrm{SU}_{1}$ sends data to the destination $\mathrm{SU}_{2}$ with the help of multiple intermediate relaying nodes $\left(\mathrm{R}_{1}, \mathrm{R}_{2}, \cdots, \mathrm{R}_{N}\right)$. The transmit powers of $\mathrm{SU}_{1}$ and the relays are strictly limited by the average tolerable interference powers at $\mathrm{PU}_{1}$ and $\mathrm{PU}_{2}$, respectively. This model can be extensively applied in cellular systems

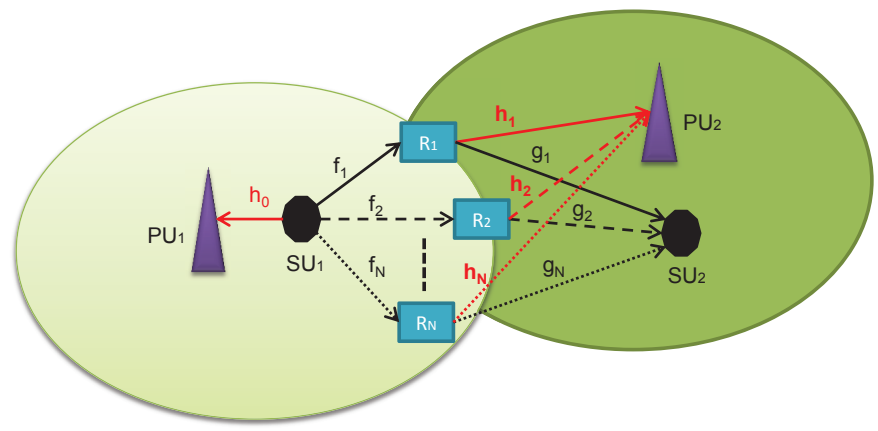

Fig. 1. System model of spectrum-sharing cooperative relaying with $N$ AF relays, where the red arrow lines refer to the interference channels and the black arrow lines stand for the desired channels.

where the base stations at two adjacent cells constitute the access points of primary users, and secondary users in each cell attempt to use the spectrum resources originally licensed to primary users in an opportunistic way through the help of other secondary nodes serving as relays in a cooperative fashion. For convenience, the base stations are also referred to as primary users. In future cellular systems, the secondary users cannot only communicate with other secondary users in the same cell but also those in adjacent cells with the help of some secondary users serving as relays. Moreover, all nodes in the system shown in Fig. 1 are equipped with single halfduplex antennas.

Unlike conventional cooperative relaying systems where the transmit power of any transmitter is generally limited by its own maximum output power, in spectrum-sharing cooperative relaying systems, secondary users can communicate with each other only when the interference power at primary users remains below tolerable levels. Hence, the transmit powers of $\mathrm{SU}_{1}$ and the relays are not only related to the desired channels $\left(\mathrm{SU}_{1}-\mathrm{R}_{i}\right.$ and $\left.\mathrm{R}_{i}-\mathrm{SU}_{2}, i=1, \cdots, N\right)$ but also to the interference channels $\left(\mathrm{SU}_{1}-\mathrm{PU}_{1}\right.$ and $\mathrm{R}_{i}-\mathrm{PU}_{2}$, $i=1, \cdots, N$ ).

The desired channels and interference channels are supposed to be independent with each other, and they are subject to block fading and remain invariant during each data transmission. The dual-hop data transmission from $\mathrm{SU}_{1}$ to $\mathrm{SU}_{2}$ are assisted by $N$ AF relays, assuming that there is no direct link between $\mathrm{SU}_{1}$ and $\mathrm{SU}_{2}$ because of deep fading. Furthermore, the data transmission between $\mathrm{SU}_{1}$ and $\mathrm{SU}_{2}$ is performed in two consecutive phases. During the first-hop phase, $\mathrm{SU}_{1}$ transmits signal $x$ with power $P_{1}$ to all relays. Accordingly, the received signal at the $i^{\text {th }}$ relay $\left(\mathrm{R}_{i}\right)$ is given by

$$
y_{i}=\sqrt{P_{1}} f_{i} x+n_{i}
$$

where $f_{i}$ denotes the complex channel coefficient between $\mathrm{SU}_{1}$ and $\mathrm{R}_{i}$, and $n_{i}$ is the additive white Gaussian noise (AWGN) at relay $\mathrm{R}_{i}$ with zero mean and variance $\sigma_{i}^{2}$. For ease of notation and without loss of generality, it is assumed throughout the rest of the paper that the AWGNs at all relays, and at $\mathrm{PU}_{1}, \mathrm{PU}_{2}$, and $\mathrm{SU}_{2}$ have the same variance $\sigma^{2}$.

During the second-hop phase, the relay $\mathrm{R}_{i}$ amplifies its received signal with power gain $\beta_{i}$ and forwards it to the 
secondary user $\mathrm{SU}_{2}$. Accordingly, the received signal at $\mathrm{SU}_{2}$ is given by

$$
y=\sqrt{P_{2}} g_{i} \beta_{i}\left(\sqrt{P_{1}} f_{i} x+n_{i}\right)+n,
$$

where $P_{2}$ denotes the transmit power at node $\mathrm{R}_{i}, g_{i}$ is the complex channel coefficient between $\mathrm{R}_{i}$ and $\mathrm{SU}_{2}$, and $n$ stands for the AWGN at $\mathrm{SU}_{2}$ with zero mean and variance $\sigma^{2}$. For mathematical tractability, the relay gain is set to $\beta_{i}=\frac{1}{\sqrt{P_{1}} f_{i}}$ [10], and substituting it into (2) yields

$$
y=\sqrt{P_{2}} g_{i} x+\frac{\sqrt{P_{2}} g_{i}}{\sqrt{P_{1}} f_{i}} n_{i}+n .
$$

Consequently, the end-to-end $\mathrm{SNR}$ from $\mathrm{SU}_{1}$ to $\mathrm{SU}_{2}$ via the relay $\mathrm{R}_{i}$ is given by

$$
\gamma_{i}=\frac{P_{2}\left|g_{i}\right|^{2}}{\frac{P_{2}\left|g_{i}\right|^{2}}{P_{1}\left|f_{i}\right|^{2}} \sigma^{2}+\sigma^{2}}=\frac{\gamma_{1 i} \gamma_{2 i}}{\gamma_{1 i}+\gamma_{2 i}},
$$

where the symbol $|x|$ stands for the amplitude of $x$, and where $\gamma_{1 i} \triangleq \frac{P_{1}}{\sigma^{2}}\left|f_{i}\right|^{2}$ and $\gamma_{2 i} \triangleq \frac{P_{2}}{\sigma^{2}}\left|g_{i}\right|^{2}$ denote the SNRs at the first hop $\left(\mathrm{SU}_{1}-\mathrm{R}_{i}\right)$ and at the second hop $\left(\mathrm{R}_{i}-\mathrm{SU}_{2}\right)$, respectively.

\section{Criteria for Power Allocation And Relay SELECTION}

In this section, the criteria for optimal transmit power allocation (at either the secondary source $\mathrm{SU}_{1}$ or the relaying node $\mathrm{R}_{i}$ ) and relay selection are established.

\section{A. Criteria for Power Allocation}

Assume that the average tolerable interference power at $\mathrm{PU}_{1}$ is $W_{1}$. In order to achieve the channel capacity $C_{1}$ at the $\mathrm{SU}_{1}-\mathrm{R}_{i}$ link, the optimal transmit power $P_{1}$ at $\mathrm{SU}_{1}$ is determined as the solution to the optimization problem:

$$
\begin{aligned}
C_{1}= & \max _{P_{1} \geq 0} \mathcal{E}_{P_{1}, f_{i}}\left\{\log _{2}\left(1+\frac{P_{1}\left|f_{i}\right|^{2}}{\sigma^{2}}\right)\right\} \\
& \text { s.t. } \quad \mathcal{E}_{P_{1}, h_{0}}\left\{P_{1}\left|h_{0}\right|^{2}\right\} \leq W_{1} .
\end{aligned}
$$

where the operator $\mathcal{E}\{$.$\} denotes the mathematical expec-$ tation, $h_{0}$ stands for the complex channel coefficient of the interference channel from $\mathrm{SU}_{1}$ to $\mathrm{PU}_{1}$. Clearly, the transmit power $P_{1}$ at $\mathrm{SU}_{1}$ is a function of $f_{i}, h_{0}, W_{1}$, and $\sigma^{2}$.

Applying the Lagrangian optimization technique, it is straightforward to show that the optimal transmit power at $\mathrm{SU}_{1}$ for the relay $\mathrm{R}_{i}$ is given by

$$
P_{1}=\left[\frac{\lambda_{1}}{\left|h_{0}\right|^{2}}-\frac{\sigma^{2}}{\left|f_{i}\right|^{2}}\right]^{+},
$$

where the operator $[x]^{+} \triangleq \max (0, x)$ and the parameter $\lambda_{1}$ is determined by the average interference power constraint satisfying the equality in $(5 b)$, such that

$$
\mathcal{E}_{h_{0}, f_{i}}\left\{\left[\lambda_{1}-\frac{\left|h_{0}\right|^{2}}{\left|f_{i}\right|^{2}} \sigma^{2}\right]^{+}\right\}=W_{1} .
$$

Notice that the optimal power allocation in $(5 \mathrm{c})$ behaves like the well-known water-filling power allocation algorithm constrained by the maximum transmit power [11]. The parameter $\lambda_{1}$ corresponds to the so-called water-level and it varies from one fading block to another. The operator $[x]^{+}$implies that the transmit power $P_{1}$ is zero if the strength of the desired channel $\left|f_{i}\right|^{2} \leq \frac{\left|h_{0}\right|^{2}}{\lambda_{1}} \sigma^{2}$, i.e., no data will be transmitted in this case. In other words, only when $\left|f_{i}\right|^{2}>\frac{\left|h_{0}\right|^{2}}{\lambda_{1}} \sigma^{2}$ can $\mathrm{SU}_{1}$ transmit to all relays, where the lower bound of $\left|f_{i}\right|^{2}$ is proportional to the strength of the interference channel $\left|h_{0}\right|^{2}$ and inversely proportional to the power allocation parameter $\lambda_{1}$.

During the second-hop phase, the relay $\mathrm{R}_{i}$ that is chosen out of the $N$ relays forwards the received signal with optimal power $P_{2}$ (the relay-selection criteria will be discussed in the next subsection), such that it achieves the channel capacity at the $\mathrm{R}_{i}-\mathrm{SU}_{2}$ link while constrained by the average tolerable interference power $W_{2}$ at the primary user $\mathrm{PU}_{2}$. That is, $P_{2}$ satisfies the optimization problem:

$$
\begin{aligned}
C_{2}= & \max _{P_{2} \geq 0} \mathcal{E}_{P_{2}, g_{i}}\left\{\log _{2}\left(1+\frac{P_{2}\left|g_{i}\right|^{2}}{\sigma^{2}}\right)\right\} \\
& \text { s.t. } \quad \mathcal{E}_{P_{2}, h_{i}}\left\{P_{2}\left|h_{i}\right|^{2}\right\} \leq W_{2},
\end{aligned}
$$

where $h_{i}$ denotes the complex channel coefficient of the interference channel from $\mathrm{R}_{i}$ to $\mathrm{PU}_{2}$.

Using a similar approach as in the fist-hop phase, $P_{2}$ is found to be given by

$$
P_{2}=\left[\frac{\lambda_{2}}{\left|h_{i}\right|^{2}}-\frac{\sigma^{2}}{\left|g_{i}\right|^{2}}\right]^{+},
$$

where the parameter $\lambda_{2}$ is determined by

$$
\mathcal{E}_{h_{i}, g_{i}}\left\{\left[\lambda_{2}-\frac{\left|h_{i}\right|^{2}}{\left|g_{i}\right|^{2}} \sigma^{2}\right]^{+}\right\}=W_{2} .
$$

\section{B. Criteria for Relay Selection}

When there are multiple relaying nodes available to assist the communication process between secondary source and destination (i.e., the number of relays $N>1$ ), the relays need to transmit on orthogonal channels so as to avoid interference with each other. Furthermore, multi-relay transmission incurs severe interference at the primary user $\mathrm{PU}_{2}$. Therefore, the technique of relay selection is promising in practical systems to leverage multi-user diversity gain. That is, the "best" relay is chosen to cooperate between $\mathrm{SU}_{1}$ and $\mathrm{SU}_{2}$ while the other relays keep silent. In general, there are two main relay-selection strategies [12], [13]. One is partial relay selection, which is based on the single-hop SNRs. The other is opportunistic relay selection, which is based on the end-to-end SNRs. For more details on implementation issues of relay selection, the interested reader is referred to [12].

1) Partial Relay Selection: Partial relay selection consists in choosing the relay that achieves the maximum SNR either at the first hop or at the second hop. Since the end-to-end SNR is given by $\gamma_{i}=\frac{\gamma_{1 i} \gamma_{2 i}}{\gamma_{1 i}+\gamma_{2 i}}$ as shown in (4), the effects of the first-hop SNR $\gamma_{1 i}$ and the second-hop SNR $\gamma_{2 i}$ on $\gamma_{i}$ are symmetric. Therefore, taking the relay selection at the first hop as an instance, the index $\hat{i}$ of the chosen relay is determined by

$$
\hat{i}=\arg \max _{i=1, \cdots, N}\left\{\gamma_{1 i}\right\} .
$$


2) Opportunistic Relay Selection: In this technique, the relay achieving the highest end-to-end $\mathrm{SNR}$ from $\mathrm{SU}_{1}$ to $\mathrm{SU}_{2}$ is chosen. Therefore, the index $\hat{i}$ of the chosen relay is given by

$$
\hat{i}=\arg \max _{i=1, \cdots, N}\left\{\gamma_{i}\right\} .
$$

Clearly, opportunistic relay selection outperforms partial relay selection in terms of the end-to-end performance because selecting a relay based on a single hop cannot guarantee the quality of channel at the other hop. More performance comparisons between these two relay-selection strategies will be illustrated in Section V-A, and their fundamental difference on diversity order will be analyzed in Section V-C.

\section{END-TO-END SNR ANALYSIS}

In this section, we analyze the end-to-end SNR from the source $\mathrm{SU}_{1}$ to its destination $\mathrm{SU}_{2}$ via the relay $\mathrm{R}_{i}$, with the average interference power constrained by $W_{1}$ at $\mathrm{PU}_{1}$ and by $W_{2}$ at $\mathrm{PU}_{2}$. The parameters $\lambda_{1}$ in (5d) and $\lambda_{2}$ in (6d) for optimal transmit power allocation are first established and, then, the distribution functions of the end-to-end SNR are derived.

Apart from the average interference power constraints at primary users, we study the effect of interference channels on the end-to-end performance of data transmission between secondary users. To this end, it is assumed that the interference channels $\mathrm{SU}_{1}-\mathrm{PU}_{1}$ with complex channel coefficient $h_{0}$ and $\mathrm{R}_{i}-\mathrm{PU}_{2}$ with channel coefficient $h_{i}$ are subject to Nakagami$m$ fading with fading parameters $m_{0}$ and $m_{i}$, respectively. The desired channels $\mathrm{SU}_{1}-\mathrm{R}_{i}$ with channel coefficient $f_{i}$ and $\mathrm{R}_{i}-\mathrm{SU}_{2}$ with channel coefficient $g_{i}$ are assumed to experience Rayleigh fading.

\section{A. Power Allocation}

According to (5d), in order to determine the optimal power allocation parameter $\lambda_{1}$ at the first hop, we derive the probability density function (PDF) of $V_{1 i} \triangleq \frac{\left|h_{0}\right|^{2}}{\left|f_{i}\right|^{2}}$. Since $h_{0}$ is subject to Nakagami fading with parameter $m_{0}$, the PDF of $\left|h_{0}\right|^{2}$ is given by

$$
f_{\left|h_{0}\right|^{2}}(x)=\frac{m_{0}^{m_{0}}}{\Gamma\left(m_{0}\right) \bar{\gamma}^{m_{0}}} x^{m_{0}-1} \exp \left(-\frac{m_{0}}{\bar{\gamma}} x\right),
$$

where $\Gamma($.$) is the Gamma function and \bar{\gamma} \triangleq \mathcal{E}\left\{\left|h_{0}\right|^{2}\right\} \frac{E_{S}}{\sigma^{2}}$. Without loss of generality, assuming $\mathcal{E}\left\{\left|h_{0}\right|^{2}\right\}=1$ yields $\bar{\gamma}=$ $\frac{E_{S}}{\sigma^{2}}$ which denotes the average $\mathrm{SNR}$ at $\mathrm{SU}_{1}-\mathrm{PU}_{1}$ link. On the other hand, the channel $\mathrm{SU}_{1}-\mathrm{R}_{i}$ with complex channel coefficient $f_{i}$ is supposed to be Rayleigh fading with the same average SNR $\bar{\gamma}$. Accordingly, the PDF of $\left|f_{i}\right|^{2}$ is given by

$$
f_{\left|f_{i}\right|^{2}}(y)=\frac{1}{\bar{\gamma}} \exp \left(-\frac{y}{\bar{\gamma}}\right) \text {. }
$$

Conditioning on $\left|f_{i}\right|^{2}$, the PDF of $V_{1 i}$ can be given by

$$
\begin{aligned}
f_{V_{1 i}}(x) & =\int_{0}^{\infty} f_{\left.V_{1 i}|| f_{i}\right|^{2}}(x \mid y) f_{\left|f_{i}\right|^{2}}(y) \mathrm{d} y \\
& =\frac{m_{0}^{m_{0}}}{\Gamma\left(m_{0}\right) \bar{\gamma}^{m_{0}+1}} x^{m_{0}-1}
\end{aligned}
$$

$$
\begin{aligned}
& \times \int_{0}^{\infty} y^{m_{0}} \exp \left[-\frac{1}{\bar{\gamma}}\left(m_{0} x+1\right) y\right] \mathrm{d} y \\
= & x^{m_{0}-1}\left(x+\frac{1}{m_{0}}\right)^{-m_{0}-1}
\end{aligned}
$$

where [14, Eq.(3.351.3)] was used to derive (11).

Then, substituting (11) into (5d), the power allocation parameter $\lambda_{1}$ can be determined by (12) at the top of next page, where we used [14, Eq.(3.194.1)] to derive (12), with ${ }_{2} F_{1}(a, b ; c, ; x)$ being the Gaussian hypergeometric function [14, Eq.(9.100)].

For the second hop, using a similar methodology as above, we can show that the power allocation parameter $\lambda_{2}$ at the relay $\mathrm{R}_{i}$ is determined by (13) at the top of next page.

Although $\lambda_{1}$ and $\lambda_{2}$ cannot be expressed in closed-form, they can be easily obtained via (12) and (13) in numerical way since Gaussian hypergeometric function is a built-in function in popular mathematical softwares, such as Mathematica. With the resultant $\lambda_{1}$ and $\lambda_{2}$, the optimal transmit powers in $(5 \mathrm{c})$ and (6c) are readily determined, respectively. Consequently, we derive the distribution functions of the end-to-end SNR in the next subsection.

\section{B. End-to-end SNR Analysis}

Substituting (5c) into the definition of $\gamma_{1 i}$ in (4), the SNR pertaining to the first hop can be given by

$$
\gamma_{1 i}=\left[\frac{\lambda_{1}}{\sigma^{2}} \frac{\left|f_{i}\right|^{2}}{\left|h_{0}\right|^{2}}-1\right]^{+}=\left[\frac{\lambda_{1}}{\sigma^{2}} V_{1 i}^{\prime}-1\right]^{+},
$$

where $V_{1 i}^{\prime} \triangleq \frac{\left|f_{i}\right|^{2}}{\left|h_{0}\right|^{2}}=\frac{1}{V_{1 i}}$. Similar to the derivation of (11), the PDF of $V_{1 i}^{\prime}$ can be expressed as

$$
f_{V_{1 i}^{\prime}}(x)=\left(1+\frac{1}{m_{0}} x\right)^{-m_{0}-1} .
$$

Substituting (15) into (14) and performing some algebraic manipulations yields the PDF of $\gamma_{1 i}$, given by

$$
f_{\gamma_{1 i}}(\gamma)=m_{0} c_{1}\left(1+c_{1} \gamma\right)^{-m_{0}-1}, \quad \gamma \geq 0
$$

where $c_{1} \triangleq \frac{\sigma^{2}}{m_{0} \lambda_{1}}$. Then, performing Laplace transform over (16), we obtain the moment generation function (MGF) of $\frac{1}{\gamma_{1 i}}$ :

$$
\begin{aligned}
M_{\frac{1}{\gamma_{1 i}}}(s) & =m_{0} c_{1} \int_{0}^{\infty} \exp \left(-\frac{s}{\gamma}\right)\left(1+c_{1} \gamma\right)^{-m_{0}-1} \mathrm{~d} \gamma \\
& =m_{0} c_{1} \int_{0}^{\infty} \frac{\gamma^{m_{0}-1}}{\left(\gamma+c_{1}\right)^{m_{0}+1}} \exp (-s \gamma) \mathrm{d} \gamma \\
& =\Gamma\left(m_{0}+1\right) \Psi\left(m_{0}, 0 ; c_{1} s\right),
\end{aligned}
$$

where we exploited [15, vol.1, Eq.(2.3.6.9)] to derive (17) with $\Psi(a, b ; x)$ being the Tricomi confluent hypergeometric function [14, Eq.(9.210.2)]. The MGF of $\frac{1}{\gamma_{2 i}}$ can be derived in a similar way and it is given by:

$$
M_{\frac{1}{\gamma_{2 i}}}(s)=\Gamma\left(m_{i}+1\right) \Psi\left(m_{i}, 0 ; c_{2} s\right),
$$

where $c_{2} \triangleq \frac{\sigma^{2}}{m_{i} \lambda_{2}}$.

With the MGFs of $\frac{1}{\gamma_{1 i}}$ and $\frac{1}{\gamma_{2 i}}$ derived, we finally obtain the distribution functions of $\gamma_{i}$, which are summarized in the 


$$
\begin{aligned}
W_{1}= & \int_{0}^{\frac{\lambda_{1}}{\bar{\gamma} \sigma^{2}}}\left(\lambda_{1}-x \sigma^{2}\right) f_{V_{1 i}}(x) \mathrm{d} x \\
= & \lambda_{1} \int_{0}^{\frac{\lambda_{1}}{\bar{\gamma} \sigma^{2}}} x^{m_{0}-1}\left(\frac{1}{m_{0}}+x\right)^{-m_{0}-1} \mathrm{~d} x-\sigma^{2} \int_{0}^{\frac{\lambda_{1}}{\bar{\gamma} \sigma^{2}}} x^{m_{0}}\left(\frac{1}{m_{0}}+x\right)^{-m_{0}-1} \mathrm{~d} x \\
= & \lambda_{1}\left(\frac{m_{0} \lambda_{1}}{\bar{\gamma} \sigma^{2}}\right)^{m_{0}}{ }_{2} F_{1}\left(m_{0}+1, m_{0} ; m_{0}+1 ;-\frac{m_{0} \lambda_{1}}{\bar{\gamma} \sigma^{2}}\right) \\
& -\frac{\sigma^{2}}{m_{0}+1}\left(\frac{m_{0} \lambda_{1}}{\bar{\gamma} \sigma^{2}}\right)^{m_{0}+1}{ }_{2} F_{1}\left(m_{0}+1, m_{0}+1 ; m_{0}+2 ;-\frac{m_{0} \lambda_{1}}{\bar{\gamma} \sigma^{2}}\right)
\end{aligned}
$$

$$
\begin{aligned}
W_{2}= & \lambda_{2}\left(\frac{m_{i} \lambda_{2}}{\bar{\gamma} \sigma^{2}}\right)^{m_{i}}{ }_{2} F_{1}\left(m_{i}+1, m_{i} ; m_{i}+1 ;-\frac{m_{i} \lambda_{2}}{\bar{\gamma} \sigma^{2}}\right) \\
& -\frac{\sigma^{2}}{m_{i}+1}\left(\frac{m_{i} \lambda_{2}}{\bar{\gamma} \sigma^{2}}\right)^{m_{i}+1}{ }_{2} F_{1}\left(m_{i}+1, m_{i}+1 ; m_{i}+2 ;-\frac{m_{i} \lambda_{2}}{\bar{\gamma} \sigma^{2}}\right) .
\end{aligned}
$$

following theorem.

Theorem 1: With constraints on average tolerable interference power at primary users in spectrum-sharing cooperative AF relaying systems, the CDF and PDF of the end-to-end SNR between two secondary users with transmission assisted by a relay are given by

$$
F_{\gamma_{i}}(\gamma)=1-\frac{c_{02} F_{1}\left(m_{0}, m_{i} ; m_{0}+m_{i}+1 ; K_{1}(\gamma)\right)}{\left(1+c_{1} \gamma\right)^{m_{0}}\left(1+c_{2} \gamma\right)^{m_{i}}}
$$

and

$$
\begin{aligned}
f_{\gamma_{i}}(\gamma) & \\
= & \frac{c_{0}\left(c_{1} m_{0}+c_{2} m_{i}\right)+c_{0} c_{1} c_{2}\left(m_{0}+m_{i}\right) \gamma}{\left(1+c_{1} \gamma\right)^{m_{0}+1}\left(1+c_{2} \gamma\right)^{m_{i}+1}} \\
& \times{ }_{2} F_{1}\left(m_{0}, m_{i} ; m_{0}+m_{i}+1 ; K_{1}(\gamma)\right) \\
& +\frac{c_{0} c_{1} c_{2} m_{0} m_{i} \gamma\left(2+c_{1} \gamma+c_{2} \gamma\right)}{\left(m_{0}+m_{i}+1\right)\left(1+c_{1} \gamma\right)^{m_{0}+2}\left(1+c_{2} \gamma\right)^{m_{i}+2}} \\
& \times{ }_{2} F_{1}\left(m_{0}+1, m_{i}+1 ; m_{0}+m_{i}+2 ; K_{1}(\gamma)\right),
\end{aligned}
$$

respectively, where

$$
c_{0} \triangleq \frac{\Gamma\left(m_{0}+1\right) \Gamma\left(m_{i}+1\right)}{\Gamma\left(m_{0}+m_{i}+1\right)}
$$

and

$$
K_{1}(\gamma) \triangleq \frac{1+c_{1} \gamma+c_{2} \gamma}{\left(1+c_{1} \gamma\right)\left(1+c_{2} \gamma\right)}
$$

Proof: Define $\gamma^{\prime}=\frac{1}{\gamma_{1 i}}+\frac{1}{\gamma_{2 i}}$, since $\gamma_{1 i}$ and $\gamma_{2 i}$ are independent, the MGF of $\gamma^{\prime}$ is the product of the MGFs of $\frac{1}{\gamma_{1 i}}$ and $\frac{1}{\gamma_{2 i}}$. Moreover, it is clear that $\gamma_{i}=\frac{\gamma_{1 i} \gamma_{2 i}}{\gamma_{1 i}+\gamma_{2 i}}=\frac{1}{\gamma^{\prime}}$, then the CDF of $\gamma_{i}$ can be expressed as

$$
\begin{aligned}
F_{\gamma_{i}}(\gamma) & =1-F_{\gamma^{\prime}}\left(\frac{1}{\gamma}\right) \\
& =1-\left.\mathcal{L}^{-1}\left\{\frac{1}{s} M_{\frac{1}{\gamma_{1 i}}}(s) M_{\frac{1}{\gamma_{2 i}}}(s)\right\}\right|_{s=\frac{1}{\gamma}},
\end{aligned}
$$

where the operator $\mathcal{L}^{-1}\{$.$\} stands for the inverse Laplace$ transform and $\left.f(x)\right|_{x=a}$ means the value of $f(x)$ at $x=$ a. Substituting (17)-(18) into (20) and using [15, vol.5, Eq.(3.34.6.3)] as well as performing some algebraic manipulations, we obtain the CDF in (19a). Furthermore, by use of the first-order derivative of Gaussian hypergeometric function [15, vol.3, Eq.(7.2.1.10)], taking the derivative of $F_{\gamma_{i}}(\gamma)$ in (19a) with respect to $\gamma$ yields the PDF $f_{\gamma_{i}}(\gamma)$ of the end-toend SNR, as shown in (19b).

Notice that, since $K_{1}(\gamma)<1$ holds true $\forall \gamma>0$, the Gaussian series expansion of the Gaussian hypergeometric function in (19a) and (19b) converges absolutely [14, Eq.(9.102)]. Therefore, the obtained CDF and PDF expressions can be easily computed by popular mathematical softwares, such as Matlab and Mathematica. Furthermore, when $\gamma=0$, it is straightforward that $K_{1}(\gamma)=1$. Subsequently, by use of [14, Eq.(9.122.1)], we have ${ }_{2} F_{1}\left(m_{0}, m_{i} ; m_{0}+m_{i}+1 ; 1\right)=$ $\frac{1}{c_{0}}$, and substituting it into (19a) yields $\left.F_{\gamma_{i}}(\gamma)\right|_{\gamma=0}=0$, as expected.

When $m_{0}=m_{i}=1$, that is, interference channels are subject to Rayleigh fading, the CDF and PDF of the end-toend SNR can be given in simple forms as summarized in the following corollary.

Corollary 1: When $m_{0}=m_{i}=1$ and the interference limits are identical $\left(W_{1}=W_{2}\right)$, we have $c_{1}=c_{2} \triangleq c_{i}$ and $c_{0}=\frac{1}{2}$. Accordingly, the CDF in (19a) and the PDF in (19b) reduce to

$$
F_{\gamma_{i}}(\gamma)=1-\frac{1}{2}\left(1+c_{i} \gamma\right)^{-2}{ }_{2} F_{1}\left(1,1 ; 3 ; K_{2}(\gamma)\right)
$$

and

$$
\begin{aligned}
f_{\gamma_{i}}(\gamma)= & c_{i}\left(1+c_{i} \gamma\right)^{-3}{ }_{2} F_{1}\left(1,1 ; 3 ; K_{2}(\gamma)\right) \\
& +\frac{1}{3} c_{i}^{2} \gamma\left(1+c_{i} \gamma\right)^{-5}{ }_{2} F_{1}\left(2,2 ; 4 ; K_{2}(\gamma)\right)
\end{aligned}
$$

respectively, where

$$
K_{2}(\gamma) \triangleq \frac{1+2 c_{i} \gamma}{\left(1+c_{i} \gamma\right)^{2}}
$$




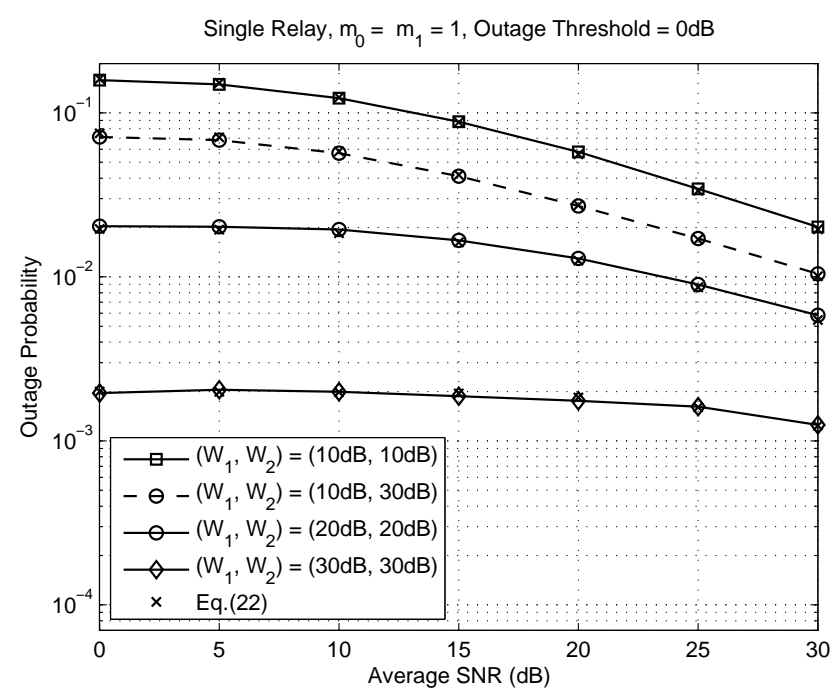

Fig. 2. Outage probability versus average SNR for different average tolerable interference powers.

\section{Performance Analysis}

In this section, based on the obtained distribution functions of the end-to-end SNR, the end-to-end performance of spectrum-sharing cooperative AF relaying systems is investigated in terms of outage probability, average symbol error rate, diversity order, and ergodic capacity.

\section{A. Outage Probability}

As an important performance measure of wireless systems, outage probability is defined as the probability that the instantaneous output SNR falls below a pre-defined threshold $\gamma_{\text {th }}$. This SNR threshold guarantees the minimum QoS requirement of secondary users. Mathematically, evaluating the CDF of the end-to-end SNR in (19a) at $\gamma=\gamma_{\text {th }}$, we immediately obtain the outage probability when there is only one relay (i.e., $i=N=1$ ). Thus, we have

$$
P_{\text {outage }}^{\text {single }}\left(\gamma_{\mathrm{th}}\right)=\operatorname{Pr}\left\{\gamma_{\text {end }}<\gamma_{\text {th }}\right\}=F_{\gamma_{i}}\left(\gamma_{\text {th }}\right) \text {, }
$$

where the superscript "single" of $P_{\text {outage }}^{\text {single }}\left(\gamma_{\mathrm{th}}\right)$ stands for the single-relay case.

On the other hand, when there are multiple relays (i.e., $N>1$ ), if the opportunistic relay-selection strategy in (8) is implemented, then according to the theory of order statistics, the CDF of the received SNR is $F_{\gamma_{i}}^{N}(\gamma)$. Accordingly, the outage probability is given by

$$
P_{\text {outage }}^{\text {multi }}\left(\gamma_{\text {th }}\right)=\operatorname{Pr}\left\{\gamma_{\text {end }}<\gamma_{\text {th }}\right\}=F_{\gamma_{i}}^{N}\left(\gamma_{\text {th }}\right),
$$

where the superscript "multi" of $P_{\text {outage }}^{\text {multi }}\left(\gamma_{\text {th }}\right)$ stands for the multi-relay case.

Figure 2 shows the outage probability versus the average SNR with different average interference power constraints. In the simulations, the outage threshold is set to $0 \mathrm{~dB}$ and interference channels are subject to Rayleigh fading. In this figure, the solid lines correspond to the cases with symmetric interference power constraints, i.e., $W_{1}=W_{2}$, and the dashed line corresponds to the non-symmetric interference power

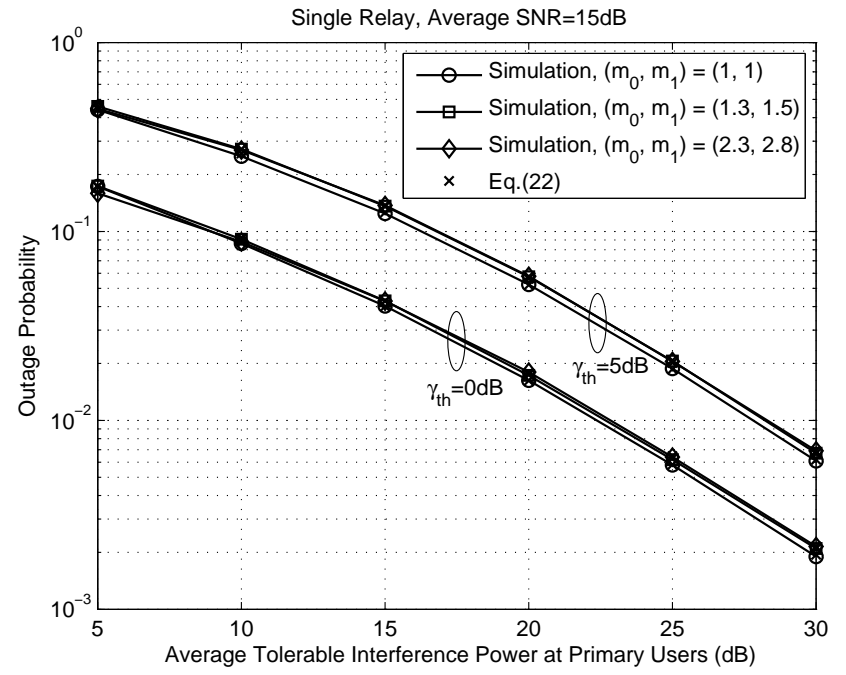

Fig. 3. Outage probability versus average tolerable interference power at primary users $\left(W_{1}=W_{2}\right)$.

constraints, i.e., $W_{1} \neq W_{2}$. It is observed that, for a given pair of interference power constraints $\left(W_{1}, W_{2}\right)$, outage probability decreases very slowly with increasing average SNR. On the other hand, when the total interference powers is fixed, for example, $W_{1}+W_{2}=40 \mathrm{~dB}$, the case with symmetric interference power constraints has lower outage probability than the case with non-symmetric constraints. This is because non-symmetric interference power constraints yield non-symmetric SNRs at consecutive hops and the end-toend SNR is dominated by the worst SNR among two hops according to (4). Furthermore, it is observed that the analytical results of (22) coincide perfectly with the simulation results.

Figure 3 depicts the outage probability versus the average tolerable interference power at primary users with fixed average SNR. In the simulations, the average interference power constraints are symmetric, that is, $W_{1}=W_{2}$. The average SNR at each link is set to $15 \mathrm{~dB}$ and the outage threshold $\gamma_{\mathrm{th}}=0,5 \mathrm{~dB}$. For a given threshold value, it is observed that outage probability decreases sharply with increasing tolerable interference power at primary users, since higher interference power limits allow higher transmit powers at the secondary source/relays. Furthermore, outage probability increases with outage threshold, as expected. On the other hand, for a given interference power limit, it is seen that outage probability varies very slightly with the fading parameters of the interference channels. When interference power limit is greater than $10 \mathrm{~dB}$, the scenario where interference channels are subject to Rayleigh fading performs slightly better than the scenario where interference channels are subject to Nakagami$m$ fading, since larger channel fluctuations in Rayleigh case leads to higher power-allocation gain [16].

Combining the observations in Fig. 2 and Fig. 3, we conclude that, 1) outage probability is dominated by the average interference power constraints and improves slowly with increasing average SNR; 2) symmetric interference power constraints lead to lower outage probability than non-symmetric constraints; 3) larger Nakagami- $m$ fading parameter on inter- 

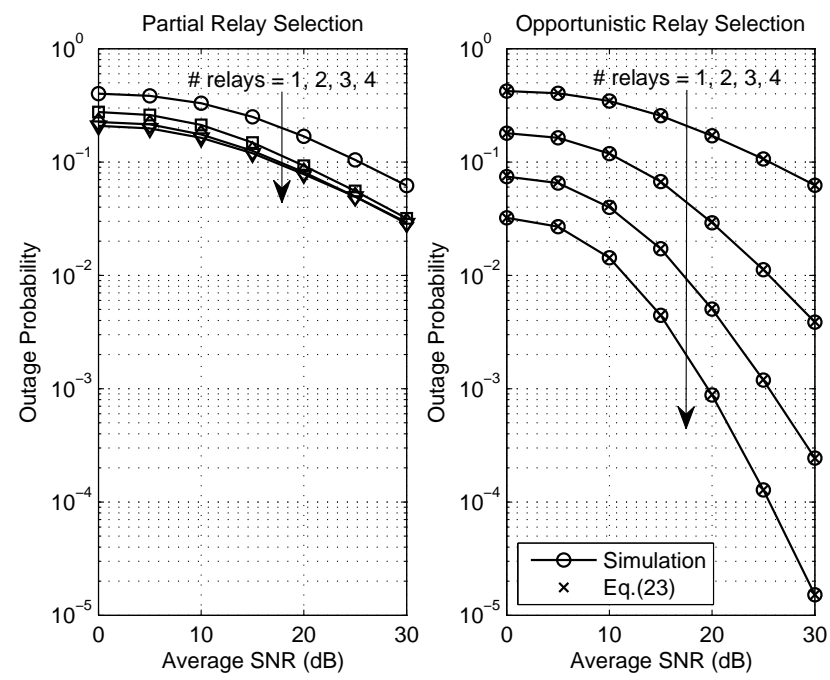

Fig. 4. Comparison of different relay-selection strategies in terms of outage probability $\left(m_{0}=m_{1}=1, W_{1}=W_{2}=10 \mathrm{~dB}\right.$ and $\left.\gamma_{\text {th }}=5 \mathrm{~dB}\right)$.

ference channels deteriorates the outage probability slightly.

Figure 4 illustrates the effect of the different relay-selection strategies discussed in Section III-B. The left-hand side panel corresponds to the partial relay selection while the righthand side panel addresses the opportunistic relay-selection technique. From the left-hand side panel, it is observed that, compared with the single-relay case, partial relay-selection strategy improves outage probability a bit when there are $N=2$ relays. However, when there are more and more relays, the outage probability improvement becomes marginal. This is because $\gamma_{i}=\frac{\gamma_{1 i} \gamma_{2 i}}{\gamma_{1 i}+\gamma_{2 i}} \approx \gamma_{2 i}$ when $\gamma_{1 i} \gg \gamma_{2 i}$, that is, the end-to-end SNR is dominated by the SNR of the worst hop. On the other hand, the right-hand side panel of Fig. 4 illustrates that opportunistic relay-selection strategy always improves the outage probability significantly when there more and more relays are available for the cooperative transmission. Furthermore, the analytical results of (23) coincide exactly with the simulation results. In particular, we observe that the outage probability of opportunistic relay selection with only $N=2$ relays outperforms partial relay selection with $N=4$ relays. The fundamental reason why opportunistic relay selection outperforms partial relay selection is its higher diversity order, which will be analytically investigated in Section V-C.

\section{B. Average Symbol Error Probability}

Average symbol error probability is one of the most commonly used performance measures of wireless communication systems. Conventionally, average symbol error probability is obtained by integrating the conditional symbol error probability $P_{s}(\gamma)$ over the PDF $f_{\gamma_{i}}(\gamma)$ of the end-to-end SNR. Mathematically, it is given by

$$
\bar{P}_{s}=\int_{0}^{\infty} P_{s}(\gamma) f_{\gamma_{i}}(\gamma) \mathrm{d} \gamma
$$

Furthermore, using the integration-by-parts method, (24) can be rewritten as

$$
\bar{P}_{s}=-\int_{0}^{\infty} P_{s}^{\prime}(\gamma) F_{\gamma_{i}}(\gamma) \mathrm{d} \gamma
$$

where $P_{s}^{\prime}(\gamma)$ denotes the first-order derivative of $P_{s}(\gamma)$ with respect to $\gamma$.

For the extensively adopted quadrature phase shift keying (QPSK) and quadrature amplitude modulation (QAM), the conditional symbol error probability is in the general form of

$$
P_{s}(\gamma)=a Q(\sqrt{b \gamma})-c Q^{2}(\sqrt{b \gamma}),
$$

where $a=2, b=1$ and $c=1$ for QPSK, and $a=4(\sqrt{M}-$ $1) / \sqrt{M}, b=3 /(M-1)$ and $c=4(\sqrt{M}-1)^{2} / M$ for $M$-QAM [17, Eqs. (5.2-59 \& 79)], and $Q(x)$ denotes the Gaussian $Q-$ function. Moreover, taking the first-order derivative of $P_{s}(\gamma)$ with respect to $\gamma$ results in

$$
P_{s}^{\prime}(\gamma)=\sqrt{\frac{b}{2 \pi}} \frac{e^{-\frac{b}{2} \gamma}}{\sqrt{\gamma}}\left[-\frac{a}{2}+c Q(\sqrt{b \gamma})\right] .
$$

Therefore, substituting the CDF in (19a) and the expression in (27) into (25), the average symbol error probability of spectrum-sharing cooperative AF relaying can be numerically evaluated.

On the other hand, it is well-known that average symbol error probability can be obtained by using the MGF of the end-to-end SNR [18]. Herein, taking the $M$-PSK constellation for instance, the average symbol error probability is given by [18, Eq.(8.23)]:

$$
\bar{P}_{s}=\frac{1}{\pi} \int_{0}^{\Theta} M_{\gamma_{i}}\left(\frac{g_{\mathrm{PSK}}}{\sin ^{2} \theta}\right) \mathrm{d} \theta,
$$

where the constants $g_{\mathrm{PSK}}=\sin ^{2}(\pi / M)$ and $\Theta=(M-$ 1) $\pi / M$. Furthermore, a closed-form approximation of the average symbol error probability can be obtained by substituting the MGF into the following expression [19, Eq.(10)]:

$$
\begin{aligned}
\bar{P}_{s} \approx & \left(\frac{\Theta}{2 \pi}-\frac{1}{6}\right) M_{\gamma_{i}}\left(g_{\mathrm{PSK}}\right)+\frac{1}{4} M_{\gamma_{i}}\left(\frac{4}{3} g_{\mathrm{PSK}}\right) \\
& +\left(\frac{\Theta}{2 \pi}-\frac{1}{4}\right) M_{\gamma_{i}}\left(\frac{g_{\mathrm{PSK}}}{\sin ^{2} \Theta}\right) .
\end{aligned}
$$

In the following, we derive the MGF of the end-to-end SNR of the system under study. We first give an integral expression in general form and, then, we derive an analytical expression for the scenario where interference channels experience Rayleigh fading.

Based on (17)-(18) and using [20, Eq.(7)], the MGF of the end-to-end SNR can be given by

$$
\begin{aligned}
\mathrm{M}_{\gamma_{i}}(s)= & 1-2 \Gamma\left(m_{0}+1\right) \Gamma\left(m_{i}+1\right) \sqrt{s} \\
& \times \int_{0}^{\infty} J_{1}(2 \beta \sqrt{s}) \Psi\left(m_{0}, 0 ; c_{1} \beta^{2}\right) \\
& \times \Psi\left(m_{i}, 0 ; c_{2} \beta^{2}\right) \mathrm{d} \beta,
\end{aligned}
$$

where $J_{1}(x)$ is the first-order Bessel function of the first kind [14, Eq.(8.402)]. Although a closed-form expression for (30) cannot be obtained, the Bessel function $\left(J_{1}\right)$ and Tricomi con- 
fluent hypergeometric function $(\Psi)$ involved in the integrand of (30) are built-in functions in popular mathematical softwares, such as Mathematica, and thus (30) can be efficiently and easily evaluated in numerical way. Furthermore, when the interference channels are subject to Rayleigh fading, based on the CDF of the end-to-end SNR in (21a), an analytical expression for the MGF can be obtained as follows.

According to the definition of the MGF of the end-to-end SNR, we have

$$
\begin{aligned}
M_{\gamma_{i}}(s) & =\int_{0}^{\infty} e^{-s \gamma} f_{\gamma_{i}}(\gamma) \mathrm{d} \gamma \\
& =s \int_{0}^{\infty} e^{-s \gamma} F_{\gamma_{i}}(\gamma) \mathrm{d} \gamma
\end{aligned}
$$

where integration-by-parts was used to derive the last equality in (31).

Then, substituting (21a) into (31) yields (32) at the top of next page, where we used the Gauss series expansion of Gaussian hypergeometric function [14, Eq.(9.100)] to derive (32). Notice that $\frac{1+2 c_{i} \gamma}{\left(1+c_{i} \gamma\right)^{2}}<1$ holds for any $\gamma>0$, thus this Gauss series expansion converges absolutely [14, Eq.(9.102)]. Furthermore, since $\frac{e^{-s \gamma}}{\left(1+c_{i} \gamma\right)^{2}}<1$ holds true $\forall \gamma>0$, by use of the comparison test, the infinite series in (32) converges absolutely. This absolute convergence allows to interchange the integration operator with the summation operator and, after performing some algebraic manipulations, (32) can be rewritten as:

$$
\begin{aligned}
M_{\gamma_{i}}(s)= & 1-s \sum_{k=0}^{\infty} \frac{1}{(k+1)(k+2)} \\
& \times \int_{0}^{\infty} \frac{\left(1+2 c_{i} \gamma\right)^{k}}{\left(1+c_{i} \gamma\right)^{2(k+1)}} e^{-s \gamma} \mathrm{d} \gamma .
\end{aligned}
$$

Applying the binomial theorem in the numerator of the integrand of (33) and performing some algebraic manipulations, we get (34) at the top of next page, where we used [15, vol.1, Eq.(2.3.6.9)] to derive (34). Finally, substituting (30) or (34) into (29), the average symbol error probability can be readily obtained.

The efficiency of above derivations is now demonstrated. Figure 5 depicts the average symbol error probability versus the average SNR, assuming QPSK constellation and $m_{0}=m_{1}=1$ in the simulations. The left-hand side panel corresponds to the single-relay case, where we observe that the analytical results of (29) match very well the simulation results. Furthermore, for a given interference power limit, the average symbol error probability decreases slowly with increasing average SNR, while for a given average SNR, the average symbol error probability decreases rapidly with increasing interference power limit.

On the other hand, the right-hand side panel of Fig. 5 illustrates the effect of opportunistic relay selection. It is seen that the average symbol error probability decreases significantly with increasing number of relays, as expected. Moreover, comparing both panels of Fig. 5, we observe that, when there are $N=3$ relays, the outage probability when opportunistic relay selection is implemented and $W_{1}=W_{2}=10 \mathrm{~dB}$ outperforms the single-relay case with $W_{1}=W_{2}=20 \mathrm{~dB}$.
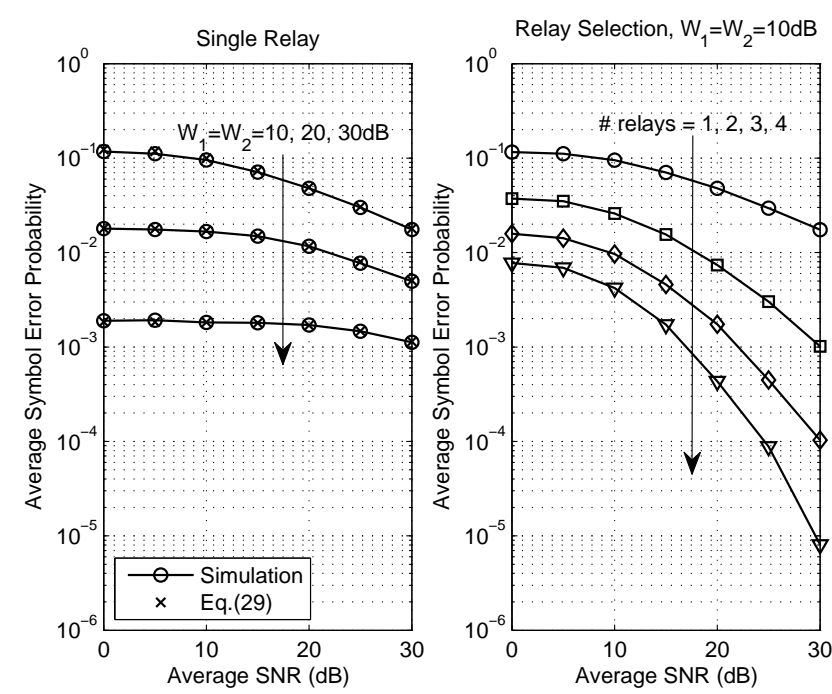

Fig. 5. Average symbol error probability versus average SNR with QPSK constellation, where interference channels are subject to Rayleigh fading.

In other words, when the interference power constraints are stringent, opportunistic relay selection can be exploited to improve the system performance significantly.

\section{Diversity Order}

In this subsection, we derive the diversity order of the system under study. Diversity order is an important parameter indicating the error probability at high SNR. According to [21, Prop. 1], if the limit of the PDF of the end-to-end SNR can be expressed as:

$$
\lim _{\gamma \rightarrow 0} f_{\gamma_{i}}(\gamma)=a \gamma^{t}+o\left(\gamma^{t+\epsilon}\right),
$$

where $a, \epsilon>0$ and $o($.$) denotes the Landau notation, then$ the diversity order $G_{d}$ is given by

$$
G_{d}=t+1 .
$$

In view of $K_{1}(\gamma)$ in (19d), it is straightforward that $\lim _{\gamma \rightarrow 0} K_{1}(\gamma)=1$. Moreover, using the series expansion of Gaussian hypergeometric function and the Gauss's theorem [14, Eq.(9.122.1)], we obtain

$$
\begin{aligned}
\lim _{\gamma \rightarrow 0}{ }_{2} F_{1}\left(m_{0}, m_{i} ; m_{0}+m_{i}+1 ; K_{1}(\gamma)\right) \\
=\lim _{\gamma \rightarrow 0} \sum_{i=0}^{\infty} \frac{\left(m_{0}\right)_{i}\left(m_{i}\right)_{i}}{i !\left(m_{0}+m_{i}+1\right)_{i}}\left[K_{1}(\gamma)\right]^{i} \\
=\frac{\Gamma\left(m_{0}+m_{i}+1\right)}{\Gamma\left(m_{0}+1\right) \Gamma\left(m_{i}+1\right)} .
\end{aligned}
$$

Then, substituting (36) into (19b), we obtain the limit of $f_{\gamma_{i}}(\gamma)$ as $\gamma \rightarrow 0$, given by

$$
\lim _{\gamma \rightarrow 0} f_{\gamma_{i}}(\gamma)=c_{1} m_{0}+c_{2} m_{i} .
$$

Finally, applying (35a)-(35b) to (37), the diversity order $G_{d}$ of spectrum-sharing system with single AF relay is given by

$$
G_{d}=1 .
$$




$$
\begin{aligned}
M_{\gamma_{i}}(s) & =s \int_{0}^{\infty} e^{-s \gamma}\left[1-\frac{1}{2}\left(1+c_{i} \gamma\right)^{-2}{ }_{2} F_{1}\left(1,1 ; 3 ; \frac{1+2 c_{i} \gamma}{\left(1+c_{i} \gamma\right)^{2}}\right)\right] \mathrm{d} \gamma \\
& =1-\frac{s}{2} \int_{0}^{\infty} \frac{e^{-s \gamma}}{\left(1+c_{i} \gamma\right)^{2}}{ }_{2} F_{1}\left(1,1 ; 3 ; \frac{1+2 c_{i} \gamma}{\left(1+c_{i} \gamma\right)^{2}}\right) \mathrm{d} \gamma \\
& =1-\frac{s}{2} \int_{0}^{\infty} \sum_{k=0}^{\infty} \frac{e^{-s \gamma}}{\left(1+c_{i} \gamma\right)^{2}} \frac{2\left(1+2 c_{i} \gamma\right)^{k}}{(k+1)(k+2)\left(1+c_{i} \gamma\right)^{2 k}} \mathrm{~d} \gamma,
\end{aligned}
$$

$$
\begin{aligned}
M_{\gamma_{i}}(s) & =1-s \sum_{k=0}^{\infty} \frac{1}{(k+1)(k+2)} \sum_{n=0}^{k}\left(\begin{array}{l}
k \\
n
\end{array}\right) 2^{n} c_{i}^{n-2(k+1)} \int_{0}^{\infty} \frac{\gamma^{n}}{\left(\gamma+\frac{1}{c_{i}}\right)^{2(k+1)}} e^{-s \gamma} \mathrm{d} \gamma \\
& =1-\frac{s}{c_{i}} \sum_{k=0}^{\infty} \frac{1}{(k+1)(k+2)} \sum_{n=0}^{k}\left(\begin{array}{l}
k \\
n
\end{array}\right) 2^{n} \Gamma(n+1) \Psi\left(n+1, n-2 k ; \frac{s}{c_{i}}\right),
\end{aligned}
$$

When there are $N>1$ relays and opportunistic relayselection strategy is implemented, it is clear that the diversity order is $N$ since the outage probability is decreased from $F_{\gamma_{i}}\left(\gamma_{\text {th }}\right)$ in the single-relay case to $F_{\gamma_{i}}^{N}\left(\gamma_{\text {th }}\right)$. The results for the diversity order being unity in the single-relay case and $N$ in the multi-relay case with opportunistic relay selection agree with the slopes of the outage probability curves shown in Fig. 2 and the right-hand panel of Fig. 4, respectively.

Notice that, although the slope of the plots of average symbol error probability versus average SNR on a log-log scale in high SNR region is usually exploited to define the diversity order, the slope of the curves of outage probability versus average SNR is also used to define the diversity order [22], [23]. Essentially, outage probability is equivalent to average symbol error probability when transmitted codewords are long enough to span multiple fading blocks [24]. This equivalence coincides with the observations that the corresponding curves have the same slopes, by comparing Fig. 2 with the lefthand panel of Fig. 5 (corresponding to the single-relay case) or by comparing the right-hand panels of Fig. 4 and Fig. 5 (corresponding to the multi-relay case with opportunistic relay selection).

On the other hand, for the partial relay selection, it has recently been proved that the diversity order is always unity no matter how many relays there are [25]. This is in agreement with the observations in the left-hand panel of Fig. 4. Therefore, based on their different diversity gains, we conclude that, when there are multiple relays available to assist the communication process between the secondary source and its destination, opportunistic relay selection is much preferable to partial relay selection.

\section{Ergodic Capacity}

Ergodic capacity is defined as the statistical mean of the instantaneous mutual information between the source and the destination, in the unit of bit/s/Hz. Mathematically,

$$
C_{\text {erg }}=\frac{1}{2} \int_{0}^{\infty} \log _{2}(1+\gamma) f_{\gamma_{i}}(\gamma) \mathrm{d} \gamma,
$$

where the factor $1 / 2$ is introduced by the fact that two transmission phases are involved in the system under study. Using the integration-by-parts method, (39) can be rewritten as (40a)-(40c) at the top of next page, where in (40a) the operator $\{f(x)\}_{a}^{b} \triangleq f(b)-f(a)$, and the CDF in (19a) was substituted into (40b) to arrive at (40c).

When there are $N$ relays and opportunistic relay-selection strategy is implemented, then based on the theory of order statistics, it is clear that the CDF of the maximum end-toend SNR is $F_{\gamma_{i}}^{N}(\gamma)$. Substituting the above CDF into (40b) and applying binomial theorem yield (41a)-(41b) at the top of next page.

Although closed-form expressions for (40c) and (41b) cannot be obtained, they can be easily evaluated in numerical way since their integrands involve only elementary functions and Gaussian hypergeometric function which is a built-in function in popular mathematical softwares. Furthermore, it is confirmed that (41b) reduces to (40c) when $N=1$. When $N>1$, (41b) implies that $C_{\mathrm{erg}}^{\text {multi }}<N C_{\mathrm{erg}}^{\text {single }}$ since the sum of finite series on its right-hand side is negative.

Equation (41b) implies that a larger number of relays results in a higher ergodic capacity, but more relays means larger amount of feedback overhead. Therefore, there is a tradeoff between capacity and feedback overhead. In order to evaluate the efficiency of each additional relay when relay selection is applied, we define the efficiency of each additional relay as the average improvement of ergodic capacity with respect to that of the single-relay case. Mathematically, it is defined as:

$$
\eta=\frac{C_{\mathrm{erg}}^{\text {multi }}-C_{\mathrm{erg}}^{\text {single }}}{(N-1) C_{\mathrm{erg}}^{\text {single }}}
$$

which will be illustrated later in Fig. 8 .

Here it is worth mentioning that a unified MGF-based approach for computing the ergodic capacity over generalized fading channels was recently developed in [26, Eq.(7)]. Un- 


$$
\begin{aligned}
C_{\mathrm{erg}}^{\text {single }} & =\frac{1}{2}\left\{\log _{2}(1+\gamma)\left[F_{\gamma_{i}}(\gamma)-1\right]\right\}_{0}^{\infty}-\frac{1}{2 \ln 2} \int_{0}^{\infty} \frac{1}{1+\gamma}\left[F_{\gamma_{i}}(\gamma)-1\right] \mathrm{d} \gamma \\
& =\frac{1}{2 \ln 2} \int_{0}^{\infty} \frac{1}{1+\gamma}\left[1-F_{\gamma_{i}}(\gamma)\right] \mathrm{d} \gamma \\
& =\frac{c_{0}}{2 \ln 2} \int_{0}^{\infty} \frac{{ }_{2} F_{1}\left(m_{0}, m_{i} ; m_{0}+m_{i}+1 ; K_{1}(\gamma)\right)}{(1+\gamma)\left(1+c_{1} \gamma\right)^{m_{0}}\left(1+c_{2} \gamma\right)^{m_{i}}} \mathrm{~d} \gamma,
\end{aligned}
$$

$$
\begin{aligned}
C_{\text {erg }}^{\text {multi }} & =\frac{1}{2 \ln 2} \int_{0}^{\infty} \frac{1}{1+\gamma}\left[1-F_{\gamma_{i}}^{N}(\gamma)\right] \mathrm{d} \gamma \\
& =\frac{1}{2 \ln 2} \sum_{n=1}^{N}\left(\begin{array}{c}
N \\
n
\end{array}\right)(-1)^{n+1} c_{0}^{n} \int_{0}^{\infty} \frac{{ }_{2} F_{1}^{n}\left(m_{0}, m_{i} ; m_{0}+m_{i}+1 ; K_{1}(\gamma)\right)}{(1+\gamma)\left(1+c_{1} \gamma\right)^{n m_{0}}\left(1+c_{2} \gamma\right)^{n m_{i}}} \mathrm{~d} \gamma \\
& =N C_{\text {erg }}^{\text {single }}+\frac{1}{2 \ln 2} \sum_{n=2}^{N}\left(\begin{array}{c}
N \\
n
\end{array}\right)(-1)^{n+1} c_{0}^{n} \int_{0}^{\infty} \frac{{ }_{2} F_{1}^{n}\left(m_{0}, m_{i} ; m_{0}+m_{i}+1 ; K_{1}(\gamma)\right)}{(1+\gamma)\left(1+c_{1} \gamma\right)^{n m_{0}}\left(1+c_{2} \gamma\right)^{n m_{i}}} \mathrm{~d} \gamma
\end{aligned}
$$

fortunately, the unified approach cannot be applied here, due to the complicated structure of the MGFs in (30) and (34), and it is expected that no closed-form capacity expression can be derived in terms of common special functions.

Figure 6 shows the ergodic capacity versus the average tolerable interference power with different fading parameters of interference channels. In the simulations, a single relay is considered, the average SNR is set to $15 \mathrm{~dB}$, and the average tolerable interference powers at primary users are supposed to be equal $\left(W_{1}=W_{2}\right)$. It is observed that, for a given interference power limit, the scenario where interference channels are subject to Rayleigh fading yields higher ergodic capacity than that of the scenario with Nakagami- $m$ fading, since larger channel fluctuations in the Rayleigh case leads to higher power-allocation gain [16]. Also, simulation results are in perfect match with the numerical results generated using (40c).

Figure 7 illustrates the ergodic capacity versus the average SNR with fixed interference power constraint. The left-hand side panel corresponds to the single-relay case. It is observed that, for a given interference power limit, the scenario where interference channels experience Rayleigh fading achieves higher ergodic capacity compared to scenarios with Nakagami$m$ fading. Furthermore, less strict interference power constraint implies higher transmit power, thus improving the ergodic capacity. On the other hand, the right-hand side panel of Fig. 7 shows the effect of opportunistic relay selection. It is seen that the ergodic capacity improves significantly with increasing number of relays, but the improvement of ergodic capacity from each additional relay becomes smaller and smaller.

Figure 8 shows the efficiency of each additional relay defined in (42). It is observed that this efficiency decreases with increasing number of relays. Furthermore, the efficiency of each additional relay is higher at low SNR than that at high SNR. Clearly, larger number of relays will lead to higher feedback overhead between the secondary destination and source nodes. Therefore, when the feedback overhead and the

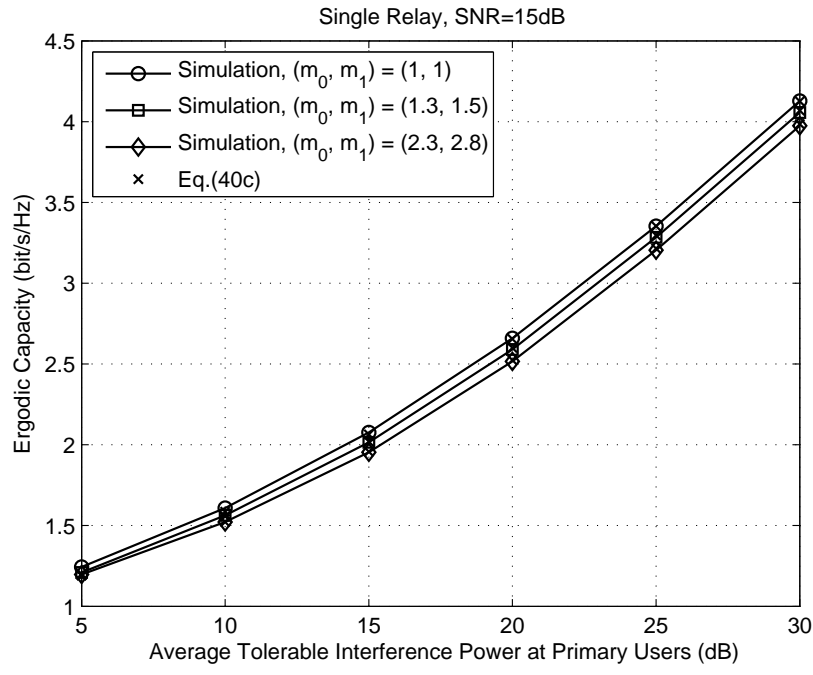

Fig. 6. Ergodic capacity versus average tolerable interference power in the single-relay case.

efficiency of each additional relay are both of concern, the scenario with two intermediate relays and opportunistic relay selection is strongly recommended in practice.

\section{CONCLUSION}

In this paper, the end-to-end performance of cooperative $\mathrm{AF}$ relaying in spectrum-sharing systems was studied. In order to obtain the optimal system performance, the transmit powers of secondary source and relays are optimized with respect to the average tolerable interference powers at primary users and the Nakagami- $m$ fading parameter of the interference channels. Furthermore, the effect of partial and opportunistic relayselection strategies on system performance were investigated. Our results show that system performance is dominated by the average tolerable interference powers, and it improves slowly with increasing average SNR and deteriorates slightly with 

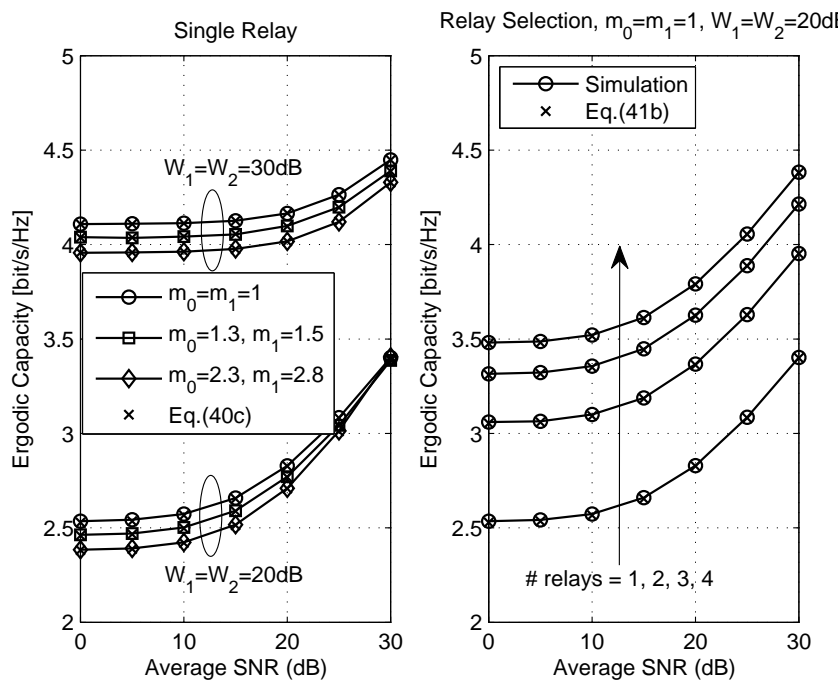

[3] M. Xia, C. Xing, Y.-C. Wu, and S. Aïssa, "Exact performance analysis of dual-hop semi-blind AF relaying over arbitrary Nakagami- $m$ fading channels," IEEE Trans. Wireless. Commun., vol. 10, no. 10, pp. 34493459, Oct. 2011.

[4] L. Musavian and S. Aïssa, "Cross-layer analysis of cognitive radio relay networks under quality of service constraints," in Proc. IEEE Veh. Tech. Conf. (VTC-S'09), Barcelona, Spain, pp. 1-5, Apr. 2009.

[5] L. Musavian, S. Aïssa, and S. Lambotharan, "Effective capacity for interference and delay constrainted cognitive radio relay channels," IEEE Trans. Wireless Commun., vol. 9, no. 5, pp. 1698-1707, May 2010.

[6] K. B. Fredj, L. Musavian, and S. Aïssa, "Closed-form expressions for the capacity of spectrum-sharing constrained relaying systems," in Proc. 17th IEEE Int. Conf. Telecomm. (ICT'10), Doha, Qatar, pp. 476-480. Apr. 2010.

[7] J. Lee, H. Wang, J. G. Andrews, and D. Hong, "Outage probability of cognitive relay networks with interference constraints," IEEE Trans. Wireless Commun., vol. 10, no. 2, pp. 390-395, Feb. 2011.

[8] V. Asghari and S. Aïssa, "Cooperative relay communication performance under spectrum-sharing resource requirements," in Proc. IEEE Int. Conf. Commun. (ICC'10), Cape Town, South Africa, pp. 1-6, May 2010.

[9] V. Asghari and S. Aïssa, "End-to-end performance of cooperative relay in spectrum-sharing systems with quality of service requirements," IEEE Trans. Veh. Technol., vol. 60, no. 6, pp. 2656-2668, June 2011.

Fig. 7. Ergodic capacity versus average SNR for different average tolerable interference powers.

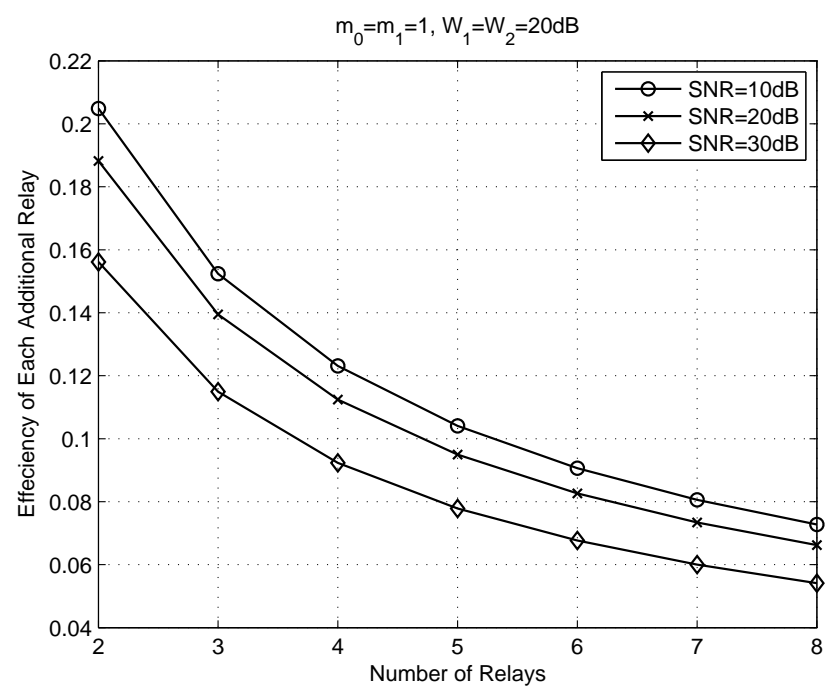

[10] M. O. Hasna and M.-S. Alouini, "End-to-end performance of transmission systems with relays over Rayleigh-fading channels," IEEE Trans. Wireless Commun., vol. 2, no. 6, pp. 1126-1131, Nov. 2003.

[11] A. J. Goldsmith and P. P. Varajya, "Capacity of fading channels with channel side information," IEEE Trans. Inf. Theory, vol. 43, no. 6 , pp. 1986-1992, Nov. 1997.

[12] A. Bletsas, A. Khisti, D. P. Reed, and A. Lippman, "A simple cooperative diversity method based on network path selection," IEEE J. Select. Area Commun., vol. 24, no. 3, pp. 659-672, Mar. 2006.

[13] A. Adinoyi, Y. Fan, H. Yanikomeroglu, H. V. Poor, and F. Al-Shaalan, "Performance of selection relaying and cooperative diversity," IEEE Trans. Wireless Commun., vol. 8, no. 12, pp. 5790-5795, Dec. 2009.

[14] I. S. Gradshteyn and I. M. Ryzhik, Table of Integrals, Series and Products, 7th Ed., Academic Press, 2007.

[15] A. P. Prudnikov, Y. A. Brychkov, and O. I. Marichev, Integrals and Series, Gordon and Breach Science Publishers, 1986.

[16] J.-P. Hong and W. Choi, "Throughput characteristics by multiuser diversity in a cognitive radio system," IEEE Trans. Signal Proces., vol. 59, no. 8, pp. 3749-3763, Aug. 2011.

[17] J. G. Proakis, Digital Communications, 4th Ed., McGraw-Hill Com., Inc., 2001.

[18] M. K. Simon and M.-S. Alouini, Digital Communication over Fading Channels, 2nd Ed., John Wiley \& Sons Inc., 2005.

[19] M. R. McKay, A. Zanella, I. B. Collings, and M. Chiani, "Error probability and SINR analysis of optimum combining in Rician fading," IEEE Trans. Commun., vol. 57, no. 3, pp. 676-687, Mar. 2009.

[20] V. Asghari, A. Maaref, and S. Aïssa, "Symbol error probability analysis for multihop relay over Nakagami fading channels," in Proc. IEEE Wireless Commun. and Networking Conf. (WCNC'10), Sydney, Australia, pp. 1-5, Apr. 2010.

larger values of the Nakagami- $m$ fading parameters pertaining to the interference channels. When the average tolerable interference powers are low, the scheme with opportunistic relay selection among two intermediate relays is recommended to improve system performance significantly without heavily increasing the feedback overhead.

\section{ACKNOWLEDGEMENT}

This study was supported in part by King Abdullah University of Science and Technology (KAUST), Thuwal, Saudi Arabia.

\section{REFERENCES}

[1] J. M. Peha, "Approaches to spectrum sharing," IEEE Commun. Mag., vol. 43, no. 2, pp. 10-12, Feb. 2005.

[2] L. Musavian and S. Aïssa, "Fundamental capacity limits of cognitive radio in fading environments with imperfect channel information," IEEE Trans. Commun., vol. 57, no. 11, pp. 3472-3480, Nov. 2009.

[21] Z. Wang and G. B. Giannakis, "A simple and general parameterization quantifying performance in fading channels," IEEE Trans. Commun., vol. 51, no. 8, pp. 1389-1398, Aug. 2003.

[22] L. Zheng and D. N. C. Tse, "Diversity and multiplexing: A fundamental tradeoff in multiple-antenna channels," IEEE Trans. Inf. Theory, vol. 49, no. 5, pp. 1073-1096, May 2003.

[23] R. U. Nabar, H. Bolcskei, and A. J. Paulraj, "Diversity and outage performance in space-time block coded Ricean MIMO channels," IEEE Trans. Wireless Commun., vol. 4, no. 5, pp. 2519-2532, Sep. 2005.

[24] H. Lee, J. G. Andrews, and E. J. Powers, "Information outage probability and diversity order of symmetric coordinate interleaved orthogonal designs," IEEE Trans. Wireless Commun., vol. 7, no. 5, pp. 1501-1506, May 2008.

[25] N. Yang, M. Elkashlan, and J. Yuan, "Impact of opportunistic scheduling on cooperative dual-hop relay networks," IEEE Trans. Commun., vol. 59, no. 3, pp. 689-694, Mar. 2011.

[26] M. D. Renzo, F. Graziosi, and F. Santucci, "Channel capacity over generalized fading channels: A novel MGF-based approach for performance analysis and design of wireless communication systems," IEEE Trans. Veh. Technol., vol. 59, no. 1, pp. 127-149, Jan. 2010. 


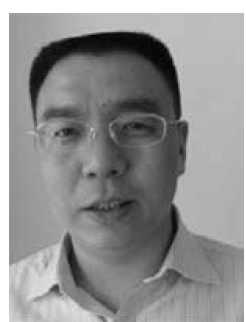

Minghua Xia obtained his Ph.D. degree in Telecommunications and Information Systems from Sun Yatsen University, Guangzhou, China, in 2007. From Mar. 2007 to July 2009, he was with the Electronics and Telecommunications Research Institute (ETRI) of South Korea, Beijing R\&D Center, Beijing, China, where he worked as a member of engineering staff and participated in the projects on the physical layer design of 3GPP LTE mobile communications. From Aug. 2009 to Feb. 2011, he was with The University of Hong Kong (HKU), Hong Kong, as a Postdoctoral Fellow. Currently, he is with King Abdullah University of Science and Technology (KAUST), Saudi Arabia. His research interests are in the area of network information theory and space-time signal processing, and in particular the design and performance analysis of multi-user multiantenna systems, cooperative relaying systems, and cognitive radio networks.

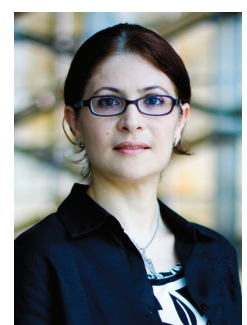

Sonia Aïssa (S'93-M'00-SM'03) received her Ph.D. degree in Electrical and Computer Engineering from McGill University, Montreal, QC, Canada, in 1998. Since then, she has been with the National Institute of Scientific Research-Energy, Materials, and Telecommunications (INRS-EMT), University of Quebec, Montreal, QC, Canada, where she is a Professor.

From 1996 to 1997, she was a Researcher with the Department of Electronics and Communications of Kyoto University, Kyoto, Japan, and with the Wireless Systems Laboratories of NTT, Kanagawa, Japan. From 1998 to 2000, she was a Research Associate at INRS-EMT, Montreal. From 2000 to 2002, while she was an Assistant Professor, she was a Principal Investigator in the major program of personal and mobile communications of the Canadian Institute for Telecommunications Research (CITR), leading research in radio resource management for code division multiple access systems. From 2004 to 2007, she was an Adjunct Professor with Concordia University, Montreal. In 2006, she was Visiting Invited Professor with the Graduate School of Informatics, Kyoto University, Kyoto, Japan. Her research interests lie in the area of wireless and mobile communications, and include radio resource management, cross-layer design and optimization, design and analysis of multiple antenna (MIMO) systems, cognitive and cooperative transmission techniques, and performance evaluation, with a focus on Cellular, Ad Hoc, and Cognitive Radio networks.

Dr. Aïssa was the Founding Chair of the Montreal Chapter IEEE Women in Engineering Society in 2004-2007, acted or is currently acting as Technical Program Leading Chair or Cochair for the Wireless Communications Symposium of the IEEE International Conference on Communications (ICC) in 2006, 2009, 2011 and 2012, as PHY/MAC Program Chair for the 2007 IEEE Wireless Communications and Networking Conference (WCNC), and as Technical Program Committee Cochair of the 2013 IEEE Vehicular Technology Conference - spring (VTC). She has served as a Guest Editor of the EURASIP Journal on Wireless Communications and Networking in 2006, and as Associate Editor of the IEEE WIRELESS COMMUNICATIONS MAGAZINE in 20062010. She is currently an Editor of the IEEE TRANSACTIONS ON WIRELESS COMMUNICATIONS, the IEEE TRANSACTIONS ON COMMUNICATIONS and the IEEE COMmunications Magazine, and Associate Editor of the Wiley Security and Communication Networks Journal. Awards and distinctions to her credit include the Quebec Government FQRNT Strategic Fellowship for Professors-Researchers in 2001-2006; the INRS-EMT Performance Award in 2004 and 2011 for outstanding achievements in research, teaching and service; the IEEE Communications Society Certificate of Appreciation in 2006-2011; and the Technical Community Service Award from the FQRNT Center for Advanced Systems and Technologies in Communications (SYTACom) in 2007. She is also co-recipient of Best Paper Awards from IEEE ISCC 2009, WPMC 2010, IEEE WCNC 2010 and IEEE ICCIT 2011; and recipient of NSERC (Natural Sciences and Engineering Research Council of Canada) Discovery Accelerator Supplement Award. 